\title{
Parent-Led Activity and Nutrition (PLAN) for Healthy Living: Design and Methods
}

\author{
William T. Dalton III, PhDa ${ }^{a}$, Karen E. Schetzina, MD, MPH ${ }^{b}$, Nicole Holt, MPH $^{c}$, Hazel Fulton- \\ Robinson, BS $^{b}$, Ai-Leng Ho, MS, RD, LDN ${ }^{b}$, Fred Tudiver, MD ${ }^{d}$, Mathew T. McBee, PhD $^{e}$, \\ and Tiejian $\mathrm{Wu}, \mathrm{MD}, \mathrm{PhD}^{\mathrm{c}, \mathrm{d}}$ \\ William T. Dalton: daltonw@etsu.edu; Karen E. Schetzina: schetzin@etsu.edu; Nicole Holt: znmh1@goldmail.etsu.edu; \\ Hazel Fulton-Robinson: robinsoh@etsu.edu; Ai-Leng Ho: HoA@msha.com; Fred Tudiver: tudiverf@etsu.edu; Mathew T. \\ McBee: matt.mcbee@unc.edu; Tiejian Wu:wut@etsu.edu \\ aDepartment of Psychology, East Tennessee State University, Johnson City, TN, USA \\ bepartment of Pediatrics, James H. Quillen College of Medicine, East Tennessee State \\ University, Johnson City, TN, USA \\ 'Department of Biostatistics and Epidemiology, East Tennessee State University, Johnson City, \\ TN, USA \\ dDepartment of Family Medicine, James H. Quillen College of Medicine, East Tennessee State \\ University, Johnson City, TN, USA \\ eFrank Porter Graham Child Development Institute, University of North Carolina Chapel Hill, \\ Chapel Hill, NC, USA
}

\section{Abstract}

Child obesity has become an important public heath concern, especially in rural areas. Primary care providers are well positioned to intervene with children and their parents, but encounter many barriers to addressing child overweight and obesity. This paper describes the design and methods of a cluster- randomized controlled trial to evaluate a parent-mediated approach utilizing physician's brief motivational interviewing and parent group sessions to treat child (ages 5-11 years) overweight and obesity in the primary care setting in Southern Appalachia. Specific aims of this pilot project will be 1) to establish a primary care based and parent-mediated childhood overweight intervention program in the primary care setting, 2) to explore the efficacy of this intervention in promoting healthier weight status and health behaviors of children, 3) to examine the acceptability and feasibility of the approach among parents and primary care providers. If proven to be effective, this approach may be an exportable model to other primary care practices.

\section{Keywords}

child; obesity; primary care; rural; treatment

\footnotetext{
(c) 2011 Elsevier Inc. All rights reserved.

Corresponding Author: William T. Dalton III, Ph.D., Assistant Professor, Address: Department of Psychology, P.O. Box 70649, East Tennessee State University, Johnson City, TN 37614, daltonw @etsu.edu, Phone: (423) 439-4465, Fax: (423) 439-5694.

Publisher's Disclaimer: This is a PDF file of an unedited manuscript that has been accepted for publication. As a service to our customers we are providing this early version of the manuscript. The manuscript will undergo copyediting, typesetting, and review of the resulting proof before it is published in its final citable form. Please note that during the production process errors may be discovered which could affect the content, and all legal disclaimers that apply to the journal pertain.
} 


\section{Introduction}

Childhood overweight and obesity has reached epidemic proportions in the United States, and has become one of the most important public health concerns today. According to the National Health and Nutrition Examination Survey, in 2003-2004 approximately 37.2\% of children ages 6-11 years old were overweight or obese [1]. Children in rural and lower socioeconomic regions, especially the Southern United States, have an even higher prevalence of obesity [2,3]. Being overweight increases the risks of developing many serious chronic health problems including hypertension, sleep apnea, and Type 2 diabetes [4,5]. Overweight children also face psychosocial problems such as poorer health related quality of life [6] and social discrimination [7].

A great deal of research has been conducted on lifestyle interventions for youth who are overweight. These programs typically target eating, physical activity, and change in other weight-related health behaviors and have revealed moderate to large effect sizes [8-10] suggesting these programs are helpful in addressing overweight. Family-based interventions are increasingly common [11] and have gained research support [12,13], especially among minority children [14]. The efficacy of family based interventions has been replicated for over 25 years [15] and recent Expert Committee recommendations [16] suggest including parents in treatment. There is little consensus on how and best to involve parents in treatment [11]. However, a recent study found group sessions with parents alone to be equally effective and more cost effective than simultaneously treating both children and parents in separate groups $[17,18]$.

Because almost all children receive their health care in a primary care setting, effective primary care delivery approaches to prevention and intervention with children who are overweight are urgently needed. Primary care physicians perceive the need to address childhood overweight as important [19] and guidelines/recommendations have been established [16]. However, primary care providers report barriers to treatment including lack of confidence and knowledge [19,20], time, materials, and reimbursement [21], and training in behavioral techniques [19]. Translating research evidence to the clinical setting has been recognized as an important research priority [12]. Studies aimed at the development of interventions for the primary care setting are needed, especially with an emphasis on acceptability and feasibility to health care providers and families. These types of programs may be especially important in rural areas characterized by high percentages of overweight [3] and limited access to health care resources [22].

\section{Objectives of Parent-Led Activity and Nutrition (PLAN) for Healthy Living}

The primary aims of the PLAN for Healthy Living pilot project are 1) to establish a primary care based and parent-mediated childhood overweight intervention program in the primary care setting, 2) to explore the efficacy of this intervention in promoting healthier weight status and health behaviors of children, 3 ) to examine the acceptability and feasibility of the approach among parents and primary care providers. The current project will include training providers to deliver a 10-week intervention with parents of overweight and obese children ages 5-11 years consisting of two individual visits utilizing brief motivational interviewing (MI) and four group sessions aimed at providing parents with the tools needed to make healthy changes in eating and physical activity behaviors in their family using the National Institutes of Health (NIH) We Can! (Ways to Enhance Children's Activity and Nutrition) Curriculum (http://www.nhlbi.nih.gov/health/public/heart/obesity/wecan/).

The primary outcome to be assessed is change in the child's Body Mass Index (BMI) percentiles represented via standardized BMI $(z B M I)$. Secondary outcomes include changes in the child's and family's eating and physical activity behaviors and the child's health 
related quality of life evaluated via parent surveys. Additionally, data will be collected on covariates as well as health care provider perceptions of treatment of child overweight and obesity. Parents and health care providers will also complete surveys and focus groups, respectively, on the acceptability and feasibility of this approach including provider perceptions of training.

At three and six months post-intervention, we hypothesize that, compared to the control clinics, in the intervention clinics: 1) children will improve more in weight status (measured by $z \mathrm{BMI}$ ), 2) parents will report more improvement in healthy eating behavior, 3 ) parents will report more improvement in physical activity behavior, and 4) parents will report more improvement in child health related quality of life. After completion of the intervention, we hypothesize that 5) parents will find the program acceptable and beneficial, and 6) providers will find the program acceptable and feasible as well as report greater competency in addressing child overweight and obesity. Analyses will also examine the roles of child and parent variables including demographic, child temperament, and parental perceptions towards child overweight, feeding attitudes and practices, dieting beliefs, and parenting style as related to primary and secondary outcomes.

\section{Study design}

\subsection{Overview}

Eighty children 5-11 years old who are overweight or obese will be recruited from two pediatric and two family practice clinics ( 20 per clinic) in Northeast Tennessee. This age range was chosen because of representativeness in family based treatment for pediatric obesity literature $[11,13]$ and the Expert Committee recommendations to involve parents of children ages $<12$ [16]. We will use cluster randomization by treating the two pediatric and two family practice clinics as pairs. We will randomly assign one clinic from each pair to the intervention or control condition. As shown in Figure 1, children will be randomly assigned to intervention and control groups based on clinic affiliation. Assignment will not be performed or revealed to providers or parents until after enrollment and consent procedures are completed.

Parents of children in the intervention group will participate in two individual visits with their clinic provider, four clinic-based group sessions moderated by a trained clinic provider, and four follow-up phone calls from the Project Coordinator or research staff. As part of the consent and baseline assessment visit, parents of children in both the intervention and control group will receive a NIH We Can! health education handbook, entitled "Families Finding the Balance: A Parent Handbook," which contains advice for parents on child eating and physical activity. This book will be provided in addition to any routine care and followup that is prescribed by their primary care provider. Both groups will participate in assessments at baseline, completion of the intervention (approximately 3 months for the control group), and 6 months post-intervention.

\subsection{Study population}

The children that will be recruited for this study are from a predominantly low socioeconomic status population residing in the Southeastern United States, specifically the region of Southern Appalachia. Whereas this area has been characterized by a particularly high prevalence of overweight and poor health behaviors [23,24], a recent study revealed only a small portion of primary care providers address these issues in practice [25]. Therefore, designing interventions to meet the needs of providers and individuals in these populations may be especially important. 


\subsection{Inclusion criteria}

Children and parents receiving care at any of the four participating primary care clinics during the enrollment period may be eligible for the study. Specific inclusion criteria will be: 1) BMI $\geq$ the $85^{\text {th }}$ percentile during the recruitment period, 2) age 5 to 11 years old during the recruitment period, and 3 ) one parent or other primary caregiver agrees to participate in the study, including individual visits and group sessions as well as phone follow-ups. Only one child per family will be included in the study.

\subsection{Exclusion criteria}

The following exclusion criteria will be applied: 1) current child or parent/primary caregiver participation in a weight management program, 2) presence of a diagnosed psychiatric/ psychological disorder in the child or parent/primary caregiver (e.g., Attention Deficit Hyperactivity Disorder, Autism, Eating Disorder), 3) presence of an underlying medical condition affecting weight status (e.g., hypothyroidism, Cushing's syndrome, chronic steroid use), 4) current dietary or physical activity restrictions (e.g., such as in children with diabetes or orthopedic problems including slipped capital femoral epiphysis), and 5) parents/ primary caregivers do not have phone accessibility. Parents/primary caregivers will also have to understand and speak English.

\subsection{Parent participation}

Parents must agree to random placement of intervention or control group. At least one parent must agree to attend the individual visits and group sessions. Children should not attend visits or sessions but childcare will be provided for extenuating circumstances.

\subsection{Recruitment and retention}

We propose to complete the enrollment within a period of seven months. To promote enrollment of study subjects, posters announcing the study and containing contact information will be prepared and posted in the 4 study clinics. Clinic staff will be trained by research staff in screening procedures and encouraged to obtain a height/weight on all 5-11 year-old children and calculate his/her BMI percentile. If the child's percentile is $285^{\text {th }}$, clinic nursing staff will begin completing a pre-screening form and provide information and/ or a brochure on the study to the family. The primary care provider will follow up by further assessing family interest in the study and for those interested, completing the pre-screening process (document whether family meets inclusion/exclusion criteria, answer questions about the study, and assess willingness of the family to be contacted by research staff). Willing families will then receive a phone call by the Project Coordinator or a research study staff to verify interest, confirm eligibility, and schedule a face to face consent visit at the participant's clinic during which baseline assessments will also be completed. Child assent will be obtained following parent consent (e.g., at parent consent visit, at a time when parent returns with child at a later date, via phone contact with parent and child). If patient volume is lower than expected, patient chart review will be conducted by the clinical staff/research team and children that meet inclusion criteria based on data from most recent clinic visits will be identified. Parents of these children will be mailed information about the study and invited to attend a joint visit with the primary care provider to confirm eligibility and assess interest in participation. Every attempt will be made to enroll minority individuals in clinics' patients.

Based on previous research, difficulties with retention are expected. A proactive approach will be taken to address these issues: 1) regular study staff meetings will be held to discuss issues that arise concerning families, 2) families will be provided with 24 hour/7 days per week contact information for the Project Coordinator, 3) families will be provided a $\$ 20$ 
incentive for participation in all assessment, individual, and group sessions (maximum $=$ $\$ 160$, intervention; $\$ 40$, control), 4) additional incentives (e.g., park admissions, t-shirts, etc.) will be offered to participants during group sessions, 5) intervention will tailor treatment to each individual family based on family characteristics and interests, 6) followup phone calls will be used to maintain contact with the control group, 7) additional followup phone calls will be used when participants in the intervention group miss a session, 8) thank you cards and birthday cards will be mailed to participants, 9) attempts will be made to schedule group sessions at times most convenient to participants, 10) a website will be developed with a section for parents that will include PowerPoint presentations from group sessions as well as schedules, 11) the Project Coordinator and research assistant will be flexible in terms of meeting families for assessment when the primary care clinic is not the most convenient location (e.g., another study clinic, child's school), 12) when applicable the Project Coordinator and research assistant will work with families to schedule a time that coincides with a previously scheduled clinic session, and 13) a total of $\$ 800$ ( $\$ 400$ in the $1^{\text {st }}$ year and $\$ 400$ in the $2^{\text {nd }}$ year) will be paid to each clinic as an incentive for the clinic's participation in the study.

\subsection{Baseline and follow-up assessments}

A schedule for data collection is presented in Table 1. During the consent and baseline assessment visit, parents will complete surveys including measures of child eating and activity behaviors, health related quality of life, and covariates of interest and assessments of children's height and weight will be obtained in both the intervention and control groups. Self administrated questionnaires will be used to collect health care provider's information. Most of the questionnaires used in the study have been validated in previous studies and some have been shortened to meet the time demands in primary care settings.

Follow-up assessment of parent reports of eating and activity behaviors, health related quality of life, and covariates of interest as well as children's height and weight will be conducted at the completion of the intervention (approximately 3 months for the control group) and 6 months after the intervention. In an effort to assess accessibility and feasibility from the parents' perspective, parents in the intervention group will also be asked to complete two NIH We Can! surveys (described below). Providers will be invited to participate in focus groups to assess program feasibility and acceptability as well as competency in treating child overweight.

\subsubsection{Primary and secondary outcome measures}

Anthropometrics: Height and weight will be assessed for the child using a standardized procedure [26]. Height will be measured with the child's shoes removed and recorded to the nearest $0.1 \mathrm{~cm}$. Weight will be measured on a calibrated scale to the nearest $0.1 \mathrm{~kg}$ with the child's shoes removed and pockets emptied. Both measurements will be taken in triplicate to ensure accuracy. Body mass index [weight in $\mathrm{kg} /(\text { height in } \mathrm{m})^{2}$ ] will be calculated and plotted on sex-and-age-specific 2000 Centers for Disease Control and Prevention growth charts [27] to determine percentile scores. Additionally, $z \mathrm{BMI}$ scores will be calculated [(measured value-average value in the reference population)/standard deviation of the reference population] for statistical analyses.

Family Eating and Activity Habit Questionnaire - Revised (FEAQ-R): The FEAQ-R was designed for parents of obese children aged 6-11 years and assesses parents' and child's eating patterns [28]. The FEAQ-R contains 32 items addressing the frequency in which the parent, spouse, and child engage in physical activity, the presence and visibility of snacks in the home, eating behaviors related to hunger, and the family eating style. In this study we will itemize the snack and sweets list to gather more accurate information regarding the 
types of foods and the frequency at which these foods are available to the child. We will also expand the physical activity items to include more child centered activities (e.g., kickball, skateboarding, jumping rope, etc.).

Dietary Pattern Screen: A dietary pattern screen will be performed using 7 items of the Eating Pattern Questionnaire which was developed via support of the American Medical Association and The Robert Wood Johnson Foundation to assist health care providers in gaining dietary information [29].

Pediatric Quality of Life Inventory (PedsQL): The PedsQL is a 23 item questionnaire that measures parent's perceptions of their child's health related quality of life [30]. In addition to a total score, the PedsQL provides indicators of physical and psychosocial functioning (via summary scores) as well as emotional, social, and school functioning (via subscale scores).

\subsubsection{Covariate measures}

Demographic Information: Demographic information on the child and parent including sex, age, and ethnicity will be collected. Parents' socioeconomic status (SES) including family size, health insurance coverage, occupation and education will also be collected. Parents will also self-report any family health history of obesity, diabetes, and hypertension.

Children's Behavior Questionnaire (CBQ): The CBQ was designed to measure the temperament of children by assessing 15 dimensions of temperament [31,32]. A shortened form containing 48 items measuring subscales of anger/frustration, attentional focusing, inhibitory control, sadness, and shyness will be used in this study.

Child Feeding Questionnaire (CFQ): The Child Feeding Questionnaire (CFQ) was designed for parents' of children aged 2 to 11 years. This questionnaire assesses the parents' perceptions and concerns regarding childhood overweight, as well as child feeding attitudes and practices [33]. The CFQ contains 31 items measuring 7 factors, 4 of which pertain to the parents' perception of their own weight and child's weight (perceived responsibility for feeding the child, perceived parent weight, perceived child weight, and concern about child's weight). The remaining 3 factors pertain to the parents' child feeding attitudes and practices (child's restriction to food, pressure child to eat, and monitoring child's eating).

Dieting Beliefs Scale (DBS): The DBS is a 16 item questionnaire that assesses parental attitudes/beliefs pertaining to the degree that they feel they have control over their own weight [34]. The current study will utilize a shortened version of the questionnaire containing 10 of these items.

Raising Children Questionnaire: The raising children questionnaire was designed to measure parental discipline strategies and parenting style [35]. A shorter revised version [36] consisting of 28 items will be used in this study.

\subsubsection{Provider perceptions of treatment and acceptability and feasibility measures}

Health Care Provider Questionnaire on Childhood Obesity: The health care provider questionnaire contains 61 items assessing provider's 1) general demographic information such as gender, education, and years of practice, 2) attitudes toward managing childhood overweight, 3) perceived barriers in the treatment of overweight children, 4) perceived skill level, and 5) readiness in treating child overweight in his/her practice [25, 37]. 
Focus Groups: Upon completion of the intervention, providers will be asked to participate in a 30-40 minute audio-taped discussion with up to two other providers. After obtaining informed consent, trained moderators will use a written guide with open-ended questions and queries to facilitate discussion regarding provider's perceptions on training and implementation of both individual visits and group sessions.

\subsubsection{Parent acceptability and feasibility measures}

We Can! Tell Us What You Think: This We Can! instrument is a brief survey that will be given to parents assigned to the intervention group pre and post intervention to assess parents knowledge about eating, physical activity, and screen time [38].

We Can! Energize Our Families Parent Program Participation Feedback Form: This We Can! instrument is a brief survey that assesses parent's perceptions regarding specific components of the We Can! program [39].

\section{Intervention}

\subsection{Delivery of the intervention}

Because the study goals are to establish and evaluate an approach to treating child overweight and obesity that will be feasible and acceptable in the primary care setting, we plan to partner with primary care providers to deliver the intervention. Each intervention clinic will be asked to identify $2-3$ providers who are willing to conduct the individual visits and facilitate the group sessions. Participating providers will be asked to complete at least eight hours of online and face-to-face training in delivery of the intervention (described below). Members of the investigative team will assist in scheduling these sessions, be present at each group session, and make all follow-up phone calls to parents. Specifically, the Project Coordinator will attend all group sessions and the Registered Dietician as well as the Pediatric Psychologist will attend at least one of the four group sessions.

\subsection{Parent-Mediated Intervention}

Individual visits-A schedule of intervention components is presented in Table 2. Parents of children in the intervention group will attend 2 individual visits with their clinic provider. These visits will be scheduled by the Project Coordinator or research team in collaboration with clinic office staff. Clinic providers will be provided with a folder for each patient that includes: 1) a Child Health Questionnaire for Parents developed by the investigators for parents to complete in the waiting area before the session; 2) the American Academy of Pediatrics (AAP) 15-Minute Obesity Prevention Protocol [40]; 3) an encounter form, the Pediatric Weight Management Medical Summary (available at http://www.aap.org/obesity/pdf/ PediatricWeightManagement_MedicalSummary_20091015.pdf); 4) three brief MI tools; 5) sample dialogue for using the brief MI tools; 6) the We Can! Try Tips to Eat Well and Move More handout used in the group sessions; 7) the AAP Prescription for Healthy Active Living (available at http://www.aap.org/obesity/whitehouse/index.html or http://www.aap.org/obesity/whitehouse/Rx\%20COLOR\%201\%20up\%20v2.pdf); and 8) 5210 educational brochures (available at www.letsgo.org and described by Rogers and Motyka [41]). The three brief MI tools that providers will be trained to use are cue cards for assessing importance and confidence for behavior change (i.e. Step 3 in the 15-Minute Protocol); parent values for their child, themselves, and their family; and pros and cons of behavior change (i.e. for use in Steps 2 and 4 of the 15-Minute Protocol).

During the first individual visit, which should occur during the first four weeks of the intervention, clinic providers will utilize the 15-Minute Obesity Prevention Protocol [41], 
which includes components of brief motivational interviewing, to interact with the family. The protocol includes coverage of five main steps (Step 1. Assessment, Step 2. Agenda Setting, Step 3. Assess Motivation and Confidence, Step 4. Summarize and Probe Possible Changes, Step 5. Schedule Follow-Up). Throughout the encounter providers will review progress and assist families in addressing barriers to behavior change as well as revising or setting new realistic We Can! "Eating Well and Moving More" goals. In the waiting room prior to the visit parents will complete the Child Health Questionnaire for Parents, a brief survey developed by the research team to assess physical activity, sedentary behavior, eating patterns, and values as well as concerns or areas in need of assistance related to the child's health. The clinic providers may use this information to further facilitate discussion during the session. Providers will also be provided with the "Pediatric Weight Management Medical Summary" and "Prescription for Healthy Active Living" developed and recommended by the AAP. The medical summary form will be provided for ease of documenting the session whereas the prescription (to be completed and signed by both the clinic provider and parent at the conclusion of the visit) will assist in individualizing an action plan and reinforcing collaboration between the parent and clinic provider.

The second individual visit, which should occur after the completion of all four group sessions, will proceed similarly to the first individual visit. Exceptions include incorporating a discussion on relapse prevention utilizing We Can! "Help Children Maintain a Healthy Weight for Life" handout as part of Step 4 (i.e., Summarize and Probe Possible Changes) of the 15-Minute Obesity Prevention Protocol. Additionally, clinic providers will be instructed to remind parents of the final schedule regarding follow-up assessments with PLAN as well as inform parents that their clinic care would return to routine care.

Group sessions-We plan to use the four visit NIH We Can! Energize our Families Curriculum available on the website (http://www.nhlbi.nih.gov/health/public/heart/obesity/wecan/) as the curriculum for the group sessions. We Can! is a recent collaboration of four Institutes of the NIH. The program uses a family-based approach aimed at helping children ages 8-13 years to maintain a healthy weight and has gained national popularity. The curriculum focuses on the concept of energy balance, and approaches to eating healthy, getting enough physical activity, and reducing screen time. Each session is designed to last about one and a half hours and will take place every other week at their primary care office. The basic format of each session includes a warm-up when participants share their experiences since the last session, a presentation of didactic information, group activities to emphasize learning points, stretch and snack breaks for modeling health behaviors, and a closing segment during which time participants identify a new tip for eating healthier and a new tip for increasing physical activity or reducing screen time for the family to try before the next session. Parent participants will receive copies of the We Can! Parent Handbook and Family Guide and will be directed to the We Can! website to access more information and resources. We have gained preliminary support for the We Can! Curriculum based on our recent pilot study of parent group sessions utilizing the curriculum [42].

Phone-follow up-Follow-up phone calls to parents will be made by the Project Coordinator or Registered Dietician. The follow-up phone call will be used to assess and reinforce progress by assessing progress on We Can!Eating Well and Moving More goals, addressing potential barriers and if needed, modifying goals, answering questions, reviewing advice and skills discussed in group sessions, and expressing encouragement and support. These calls also provide an opportunity to individualize advice and recommendations utilizing a brief MI approach as well as offer reminders of upcoming activities related to the project. The Registered Dietician will make at least one of follow-up phone calls to each intervention participant. Documentation of the calls, including parents' progress towards 
meeting their goals, will be maintained in each child's study folder in the clinic for review by the primary care provider prior to the individual visits.

\subsection{Control Condition}

Families assigned to the control condition will complete assessments at baseline, 3-months, and 6-months. As noted previously, parents will receive "Families Finding the Balance: A Parent Handbook," a health education handbook adopted by NIH We Can! during the baseline assessment. However, no intervention will be offered to this group but rather routine care and follow-up that is typically prescribed by their clinic provider.

\subsection{Provider Training in the Intervention}

Individual visit training-Online training for the individual visits will consist of viewing two narrated Power Point presentations (available at www.etsu.edulcom/plan) that were created by the investigators to provide overviews of 1) the PLAN study rationale, methods, and procedures, and 2) the characteristics, guiding principles, and foundational clinical skills of brief motivational interviewing. After viewing the online presentations providers will be asked to complete an 8-item quiz to assess their understanding of brief MI. Correct responses will be provided electronically after the quiz is submitted to the investigators. During the face-to-face training, material covered in the online training will be reviewed and expanded upon and a videotaped encounter demonstrating the use of brief MI to counsel a parent of an overweight child with unhealthy eating behaviors [43] will be viewed and discussed. Two individual visit scenarios following the AAP 15-Minute Obesity Prevention Protocol and using brief MI will be practiced and discussed. During the face-to-face sessions providers will also be provided with copies of the AAP Pediatric Obesity Clinical Support Decision Chart 5210 (available at https://www.nfaap.org/netFORUM/eweb/DynamicPage.aspx? webcode=aapbks_productdetail \&key=3ffed110-2471-40f3-9547-61666fa5b6ed) and a sample of the study patient folder that will be used during the individual visits. In an effort to support provider adherence to training, study staff will engage in regular contact with providers to assist in addressing concerns/barriers to completing individual visits.

Group session training-Providers facilitating the group sessions will be asked to view the 9-chapter online training provided on the We Can! website for group leaders (available in PowerPoint, plain text, and Flash versions at http://www.nhlbi.nih.gov/health/public/heart/obesity/wecan/partner-with-us/onlinetraining.htm\#ViewTraining). In addition, they will be provided with a copy of "Parent Program: A Leader's Guide" that is also available from the We Can! website. Finally, to make it easier to present the material covered in the curriculum, providers will be provided a pre-recorded narrated Power Point covering the didactic information and a digital video of the stretch break to display during each of the four sessions. During the face-to-face training, the providers' role in facilitating the sessions and communication skills will be discussed following the basic outline common to each group session. Use of the pre-recorded presentations and activity materials (provided by the research team) used in each session will be demonstrated. Finally, the role of study staff in scheduling and setting up for each session will be clarified. Presence of the study staff at each group session will help to ensure adherence to the group session curriculum and allow questions to be addressed.

Phone follow-up training-The Project Coordinator and Registered Dietician will be trained in the use of brief MI during phone calls made to parents following each of the group sessions. Training will consist of viewing and discussing a video [43] of a phone call with the parent of an overweight child. In addition, they will be trained in the use of a 
standardized script. During weekly communication among the project team members, experience with making the phone calls and any questions that may arise will be addressed.

\section{Statistical Considerations}

\subsection{Statistical analyses}

The models utilized to answer our research questions will be analysis of covariance models (ANCOVA). Analysis of covariance will be chosen because it is the most powerful of the pretest-posttest designs and it produces unbiased estimates of treatment effects when treatment is randomly assigned [44]. The ANCOVA model will regress the posttest score on a pretest score, an indicator of treatment, and (typically) a set of covariates. The model will take the following form:

$$
\begin{gathered}
Y_{i}=b_{0}+b_{1} \text { pretest }_{i}+b_{2} \text { tx }_{i}+\sum_{j=1}^{k} b_{j} \text { covariate }_{j}+e_{i} \\
e_{i} \sim N\left(0, \sigma^{2}\right)
\end{gathered}
$$

where the subscript $i$ indexes children, $k$ the total number of covariates, $j$ the $j$ th covariate. The outcome, which varies by research question, is represented by $Y$. The model parameter $b_{0}$ represents the intercept, $b_{1}$ represents the influence of the pretest, $b_{2}$ the effect of treatment, and $b_{3}$ through $b_{k}$ the effects of the covariates. The residual, represented by $e_{i}$, it is assumed to be normally distributed with mean zero and constant variance sigma squared. For all research questions, the test of the treatment effect will be represented as the hypothesis test of parameter $b_{2} ; b_{2}$ is hypothesized to be negative for RQ1 and positive for RQ2-RQ4. Statistical significance will be defined as $p<.05$ via a two-sided hypothesis test. Confidence intervals (95\%) and Cohen's $d$ effect sizes will be reported.

Covariates-A set of covariates will be included in the model in order to increase power by reducing residual variance in the outcome. Covariates will include child and parent sex, age, and ethnicity, family SES, family health history, and parent scores on the CBQ, CFQ, DBS, and Raising Children Questionnaire.

In this study, children will be sampled from clinics. Ordinarily, this cluster randomized design would require a multilevel or random effects model in order to account for the nonindependence of children sampled from the same clinic, because ordinary statistical models (such as the proposed ANCOVA) assume that residuals are independent and identically distributed (i.i.d; [45]). Data from cluster randomized trials typically violate this assumption of independence and therefore require specialized methods in order to avoid misestimation of standard errors and increased risk of Type-I errors. However, in this study, there will be only four centers. Random effects models, which correctly account for non-independence, do so by estimating an additional variance component to represent the within-cluster variance, but four centers are inadequate for stable estimation of the cluster-level variance component [46]. Therefore, a fixed effects approach will be used [47]. Clinics will be dummy-coded with an indicator of each clinic entered into the model as a covariate, with one left out to define the reference clinic. The coefficients associated with the clinic dummy indicators will purge the between-centers variance from the residual, thereby ensuring that the ANCOVA model assumptions are met.

\subsection{Statistical power analysis}

Statistical power refers to the probability of rejecting the null hypothesis when it is false [48], in other words, it is the probability of detecting a treatment effect when the treatment effect actually exists. Statistical power for ANCOVA designs depend on the sample size, the effect size of the intervention, and the residual variance of the outcome. The residual 
variance, in turn, depends on the total variance of the outcome, the variance explained by the pretest, and the variance explained by the covariates. Therefore, given a priori specifications of sample size, alpha, effect size of intervention, and residual variance, power can be computed. By convention, power of $80 \%$ or more is usually considered adequate.

Because researchers are often unsure about anticipated effect sizes, it often more practical to compute the minimum detectable effect size (MDES) at $80 \%$ power given a sample size and an assumption regarding the residual variance. We took this approach for our power analysis, which is summarized in Figure 2. The figure displays the between the minimum detectable effect size at $80 \%$ power versus sample size for three different assumptions regarding the proportion of variance explained by the pretest and covariates $-50 \%, 60 \%$ and $70 \%$. The study will enroll a sample size of 80,20 from each of the four clinics. Because we anticipate some attrition, our effective sample size is likely to decrease over time. Therefore, the figure provides a plot of the MDES against the (remaining) sample size after attrition has taken place. With a $10 \%$ attrition rate, we will have 72 subjects at the three month time point and 65 subjects at the six month follow-up. Therefore, our estimates of the MDES range from 0.37 to 0.48 at the three month assessment and from 0.39 to 0.50 at the six month assessment. These effect sizes are expressed as Cohen's $d s$ and represent "moderate" effect sizes according to Cohen's (1988) guidelines [48]. We performed our power analysis using Optimal Design version 2.0 [49]. We believe that our intervention will produce effect sizes in this range based on results of previous treatment studies [8-10,12].

\subsection{Data safety monitoring plan}

Data will be collected using standardized paper forms and will only be identified with the study identification number of the participant. The record that links the name of the participant and the study identification number will be kept confidential by the Principal Investigator in a secured cabinet. Collected forms will be transported to the Principal Investigator's office. Data will be entered in the computer independently by the Project Coordinator and two research assistants, and discrepancies will be corrected by the Project Coordinator, based on source documents. The quality of the data will be monitored once per month. The Principal Investigator will analyze the data, using SPSS Statistical Software. Data quality will be monitored by random inspection of the completed forms by the research assistants and any problems detected will be discussed with the Principal Investigator. See Table 3 for a detailed plan that will be used to monitor the safety of study participants.

\subsection{Data management}

The Project Coordinator will be responsible for managing data. The Principal Investigator and Co-Investigators will supervise the Project Coordinator in setting up a computerized database. All data will be password protected and backed up on hard drive. All research staff involved in data collection will be trained in data collection procedures including those required for obtaining anthropometric measures.

\section{Discussion}

PLAN for Healthy Living is a cluster randomized clinical trial that will utilize a parentmediated approach in primary care, which will include parents or primary caregivers attending individual visits and group sessions with providers, to address child overweight and obesity. In addition to weight management, the current study aims to examine the acceptability and feasibility of the approach among parents and primary care providers. We predict that children in the intervention clinics will demonstrate weight loss or maintenance at 3 and 6-month follow-up and parents will report healthier eating habits and increased physical activity as well as health related quality of life for their child during these 
assessment periods. We also predict that at the conclusion of the intervention parents will report favorable impressions of the program and providers will report acceptability and feasibility of the approach as well as increased competency in assessing and treating child overweight and obesity in their practice.

This pilot study will provide preliminary data that may be helpful in future design of largerscale trials and is important for several reasons including 1) a need for applied and translational research in primary care settings, 2) attention to provider concerns regarding treatment efficiency and training, 3) utilization and assessment of the latest evidence ased practices/recommendations including brief MI, group sessions, the latest tools recommended by NIH and AAP and available to the public, and parents as targets of change, and 4) attention to an underserved and at-risk population. We follow with a discussion on each of these issues.

Because almost all children receive their health care in primary care settings, primary care providers are optimally positioned to intervene with patients and families in the prevention and management of childhood overweight. Numerous organizations including the AAP [50] recommend that physicians determine BMI for all children in their practices and offer appropriate interventions to those who are overweight or obese. However, many physicians do not address this issue with their patients [51,52]. In fact, studies indicate that health care providers recognize and initiate treatment for fewer than $20 \%$ of overweight children [53]. The content and quality of nutrition and physical activity interventions also vary. Primary care physicians may counsel patients on the risks of overweight and give advice to eat less and exercise more [54]; however, time constraints often prevent discussion of how to implement the eat-less, exercise-more prescription and may limit the physician's opportunity to apply principles of behavior change [55]. Other barriers to treating children who are overweight include lack of patient motivation and parent involvement, limited support services, perceived treatment futility, lack of reimbursement, and inadequate clinician knowledge and treatment skills [56]. The current program was developed with attention to several of these factors.

Restructuring the delivery of primary care using the Chronic Care Model has been shown to be effective in reducing risk for conditions such as diabetes, asthma, and cardiovascular disease [57-59] and has been advocated to address child overweight and obesity [40,60]. In the PLAN study intervention, providers will be trained in the use of decision-support tools (i.e., AAP Pediatric Obesity Clinical Support Decision Chart 5210), care delivery will be designed to ensure coordinated care by the primary care provider and registered dietician through group sessions and structured follow-up phone calls, and parents will be educated in approaches to making healthy changes in their family's eating and physical activity behaviors (i.e., self-management support).

The current project will utilize brief intervention, specifically brief MI and group sessions, in an effort to demonstrate efficiency. MI may be described as a patient-centered and goaldirected treatment approach for eliciting behavior change [61]. Patients are supported in exploring and resolving ambivalence related to current health behaviors and values as well as future goals [61]. A recent meta-analytic review [62] of randomized controlled trials using MI found significant effects across a variety of health behaviors including weight loss. Although the majority of research has been published on MI with adults the value with pediatric populations appears promising $[63,64]$. Due to its adaptability to brief formats it has been considered a brief intervention suited for pediatric health care settings [63]. Pediatric providers and training programs have even begun to incorporate MI [64]. In fact, Barlow and the Expert Committee on recommendations for the prevention, assessment, and treatment of child and adolescent overweight and obesity [40] recommend the use of 
motivational interviewing. In addition to research and clinical recommendations provided via a conceptual and evidence review of MI for pediatric obesity [65], a recent feasibility study [66] found that targeting children via parents attending 1-2 sessions with a pediatrician or a pediatrician and dietician, respectively, found MI to be a promising approach for preventing child obesity. The current study will utilize the 15-minute obesity prevention protocol [40], which incorporates elements of MI, to facilitate discussions with parents in individual visits.

Group intervention may provide participants an opportunity to benefit through active participation, observation, and collaboratively working through individual concerns in a supportive, confidential environment [67]. A study with adults by Renjilian and colleagues [68] examined individual versus group therapy for overweight and found that group treatment resulted in greater weight loss. However, the American Dietetic Association recently reviewed group sessions versus individual visits in terms of effectiveness of treating childhood overweight [69]. It identified three studies and concluded that within multicomponent childhood overweight treatment programs, limited evidence suggests that group versus individual treatment formats have similar effects on improving adiposity outcomes. The Institute of Medicine recently identified key approaches for improving the delivery of primary health care for patients [60]. Group visits were suggested to provide a cost-effective approach for treating many important health problems by offering ample time for education and discussion [70]. This study will provide the opportunity to examine both the effectiveness and feasibility of utilizing groups in the treatment of overweight treatment in the primary care setting.

Based on research documenting lack of training, materials, and skills in behavioral techniques [19-21], the current study will also provide training in brief MI and group sessions to increase provider competency and skills for addressing child overweight. Providers will complete at least 8 hours of online and face-to-face training in brief intervention, MI, and delivery of group sessions. Providers will receive continuing education credit as well as monetary incentives for participation in training. At the conclusion of the study, we plan to conduct focus groups with providers to evaluate perceptions regarding acceptability and feasibility including the time and quality related to training. Due to the emphasis on acceptability and feasibility we will provide support (i.e., training, answering questions via phone or scheduled meetings) for the individual visits and assistance (i.e., staff presence at group sessions to monitor adherence to the We Can! protocol and answer questions) but will not serve as interventionists. Thus, we aim to address concerns regarding treatment integrity although this should be considered when interpreting the findings.

In addition to incorporating the latest evidence based tools recommended by the NIH and AAP as well as other organizations, the current study utilizes parents as the target of change. Parents have been recognized as influential in the development of eating and physical activity patterns [71,72] and parental involvement has been recommended as a component of treatment of child overweight and obesity [16]. A Cochrane systematic review of childhood overweight treatment interventions last updated in May 2004 examined 18 randomized controlled trials with a total of 975 participants [73]. Among their conclusions the authors stated that "there may be some additional benefit to behavior therapy when the parents, rather than the child, are given the primary responsibility for behavior change." The intervention that we propose to test in this study is based on a behavioral approach in which the parent, rather than the child, is given the primary responsibility for behavior change. Exclusively targeting the parent contributes to the applicability to the primary care setting, a venue in which parent education and anticipatory guidance are delivered regularly. Because this approach limits direct intervention with children, it may limit children's perception of being "an overweight patient" and result in fewer potentially adverse psychological effects 
as well as less resistance. Recent studies also suggest a parent only intervention may be costeffective in treating child overweight and obesity [18]. However, this parent-mediated approach needs to be tested in the primary care setting.

Participants for the current study will be recruited from the Southern United States, specifically Southern Appalachia. Our study population is of lower socioeconomic status than those in previous studies and resides within the Appalachian region of the Southeastern United States, an area with high rates of overweight in which few studies have been conducted. Among all states, Tennessee, which is the proposed location for the current study, has the $5^{\text {th }}$ highest rate in adult overweight and $2^{\text {nd }}$ highest rate of overweight among high school students [74]. Thus, our study will make valuable progress in determining not only the utility and feasibility of overweight interventions in primary care but also with attention to a population with health disparities.

In summary, the primary care setting is an ideal venue in which to reach a large number of children and their families and a well-positioned setting to identify those in need of lifestyle interventions. If successful, this pilot project utilizing the partnership of primary care providers with parents and the use of brief intervention techniques and the latest recommended NIH and AAP tools can provide evidence for a practical and powerful approach toward reducing child overweight even among high risk populations. Being that PLAN is a family based approach the project may also increase parent's knowledge of nutrition and physical activity and contribute to healthy lifestyle changes in parents thus having potential far reaching effects. The current report provides information to facilitate replication in different patient populations and larger-scale trials as well as evidence based assessment and intervention practice in the clinical setting.

\section{Acknowledgments}

This project was funded by the National Institutes of Health (1R15HD054950-01A2).

\section{References}

1. Ogden CL, Carroll MD, Curtin CR, McDowell MA, Tabak CJ, Flegal KM. Prevalence of Overweight and Obesity in the United States. JAMA. 2006; 295:1549-1555. [PubMed: 16595758]

2. Institute of Medicine. [Accessed March 24, 2010] Report: Preventing Childhood Obesity: Health in the Balance. 2004. http://www.iom.edu/Reports/2004/Preventing-Childhood-Obesity-Health-in-the-Balance.aspx

3. Lutfiyya MN, Lipsky MS, Wisdom-Behounek J, Inpanbutr-Martinkus M. Is rural residency a risk factor for overweight and obesity for U.S. children? Obesity. 2007; 15:2348-2356. [PubMed: 17890504]

4. Dietz WH. Health Consequences of Obesity in Youth: Childhood Predictors of Adult Disease. Pediatrics. 1998; 101:518-525. [PubMed: 12224658]

5. Marcovecchio M, Mohn A, Chiarelli F. Type 2 diabetes mellitus in children and adolescents. J Endocrinol Invest. 2005; 28:853-863. [PubMed: 16370570]

6. Tsiros MD, Olds T, Buckley JD, Grimshaw P, Brennan L, Walkley J, Hills AP, Howe PR, Coates AM. Health-related Quality of Life in Obese Children and Adolescents. Int J Obes (Lond). 2009; 33:387-400. [PubMed: 19255583]

7. Puhl RM, Latner JD. Stigma, obesity and the health of the nation's children. Psychol Bull. 2007; 133:557-580. [PubMed: 17592956]

8. Haddock CH, Shadish WR, Klesges RC, Stein RJ. Treatment for childhood and adolescent obesity. Ann Behav Med. 1994; 16:235-244.

9. Snethen JA, Broome ME, Cashin SE. Effective weight loss for overweight children: A meta analysis of intervention studies. J Pediatr Nurs. 1996; 21:45-56. [PubMed: 16428013] 
10. Wilfley DE, Tibbs TL, Van Buren DJ, Reach KP, Walker MS, Epstein LH. Lifestyle Interventions in the Treatment of Childhood Overweight: A Meta-Analytic Review of Randomized Controlled Trials. Health Psychol. 2007; 26:521-532. [PubMed: 17845100]

11. Kitzmann KM, Beech BM. Family-based interventions for pediatric obesity: methodological and conceptual challenges from family psychology. J Fam Psychol. 2006; 20:175-189. [PubMed: 16756393]

12. Kitzmann KM, Dalton WT III, Stanley CM, et al. Lifestyle interventions for youth who are overweight: A meta-analytic review. Health Psychol. 2010; 29:91-101. [PubMed: 20063940]

13. Young KM, Northern JJ, Lister KM, Drummond JA, O'Brien WH. A meta-analysis of family behavioral weight-loss treatments for children. Clin Psychol Rev. 2007; 27:240-249. [PubMed: 17070638]

14. Seo DC, Sa J. A meta-analysis of obesity interventions among U.S. minority children. Journal of Adolescent Health. 2010; 46:309-323. [PubMed: 20307819]

15. Epstein LH, Paluch RA, Roemmich JN, Beecher MD. Family-based obesity treatment, then and now: Twenty-five years of pediatric obesity treatment. Health Psychol. 2007; 26:381-391. [PubMed: 17605557]

16. Spear BA, Barlow SE, Ervin C, Ludwig DS, Saelens BE, Schetzina KE, et al. Recommendations for treatment of child and adolescent overweight and obesity. Pediatrics. 2007; 120:S254-S288. [PubMed: 18055654]

17. Janicke DM, Sallinen BJ, Perri MG, Lutes LD, Hureta M, Silverstein JH, et al. Comparison of parent only vs family based interventions for overweight children in underserved rural settings: outcomes from Project Story. Arch Pediatr Adolesc Med. 2008; 162:1119-1125. [PubMed: 19047538]

18. Janicke DM, Sallinen BJ, Perri MG, Lutes LD, Silverstein JH, Brumback B. Comparison of program costs for parent only and family based interventions for pediatric obesity in medically underserved rural settings. J Rural Health. 2009; 25:326-330. [PubMed: 19566621]

19. Holt NM, Schetzina KE, Dalton WT III, Tudiver F, Fulton Robinson H, Wu T. Primary care practice addressing child overweight and obesity: A survey of primary care physicians at four clinics in Southern Appalachia. South Med J. 2011; 104(1):14-19. [PubMed: 21119559]

20. Huang J, Yu H, Marin E, Brock S, Carden D, Davis T. Physicians' weight loss counseling in two public hospital primary care clinics. Acad Med. 200; 79:156-161. [PubMed: 14744717]

21. Kushner RF. Barriers to providing nutrition counseling by physicians: a survey of primary care practitioners. Prev Med. 1995; 24:546-552. [PubMed: 8610076]

22. Jones, CA.; Parker, TS.; Ahearn, M.; Mishra, AK.; Variyam, JN. Economic Research Service. Bulletin Number 57. United States Department of Agriculture; Health status and health care access of farm and rural populations; p. 1-72.Retrieved from http://www.ers.usda.gov/publications/eib57/

23. U.S. Department of Health and Human Services, Health Resources and Service Administration. The National Survey of Children's Health Overweight and Physical Activity among Children: A Portrait of States and the Nation. 2005. p. MCH00202

24. Centers for Disease Control and Prevention. Estimated County-Level Prevalence of Diabetes and Obesity- United States, 2007. MMWR. 2009; 58:1259-1263. [PubMed: 19940830]

25. Wu T, Tudiver F, Wilson JL, Velasco J. Child obesity interventions in rural primary care practice: A survey of primary care providers in Southern Appalachia. South Med J. 2007; 100:1099-1104. [PubMed: 17984741]

26. Frisancho, AR. Anthropometric Standards for the Assessment of Growth and Nutritional Status. University of Michigan Press; Ann Arbor, MI: 1990.

27. Kuczmarski RJ, Ogden CL, Guo SS, Grummer-Strawn LM, Flegal KM, Mei Z, et al. 2000 CDC growth charts for the United State: Methods and development. Vital Health Statistics. 2002; 246:1-190.

28. Golan M, Weizman A. Reliability and validity of the family eating and activity habits questionnaire. Eur J Clin Nutr. 1998; 52:771-777. [PubMed: 9805227]

29. Kushner, RF. Roadmaps for Clinical Practice: Case Studies in Disease Prevention and Health Promotion-Assessment and Management of Adult Obesity: A Primer for Physicians. Chicago, Illinois: American Medical Association; 2003. 
30. Varni JW, Limbers CA, Burwinkle T. Parent proxy-report of their children's health-related quality of life: An analysis of 13,878 parents' reliability and validity across age subgroups using the PedsQL 4.0 Generic Core Scales. Health Qual Life Outcomes. 2007; 5:110.10.1186/1477-7525-5-2 [PubMed: 17201920]

31. Putnam SP, Rothbart MK. Development of Short and Very Short forms of the Children's Behavior Questionnaire. J Pers Assess. 2006; 87:103-113.

32. Rothbart MK, Ahadi SA, Hershey KL, Fisher P. Investigations of temperament at 3-7 years: The Children's Behavior Questionnaire. Child Development. 2001; 72:1394-1408. [PubMed: 11699677]

33. Birch LL, et al. Confirmatory factor analysis of the Child Feeding Questionnaire: A measure of parental attitudes, beliefs and practices about child feeding and obesity proneness. Appetite. 2001; 6:201-210. [PubMed: 11358344]

34. Stotland S, Zuroff DC. A new measure of weight locus of control: The Dieting Beliefs Scale. J Pers Assess. 1990; 54:191-203. [PubMed: 2313541]

35. Greenberger E, Goldberg W. Work, Parenting, and the Socialization of Children. Dev Psychol. $1989 ; 25: 22-35$.

36. Shumow L, Vandell DL, Posner JK. Harsh, firm, and permissive parenting in low-income families: Relations to children's academic achievement and behavioral adjustment. J Fam Issues. 1998; 19:483-507.

37. Story MT, Neumark-Stzainer DR, Sherwood NE, et al. Management of Child and Adolescent Obesity: Attitudes, Barriers, Skills, and Training Needs Among Health Care Professionals. Pediatrics. 2002; 110:210-214. [PubMed: 12093997]

38. National Institutes of Health. NIH Publication No. 07-5850. 2008. Tell Us What You Think: We Can! Energize our Families Parent Program: A Leaders Guide.

39. National Institutes of Health. NIH Publication No. 07-5839. 2008. Parent Program Participant Feed Back Form: We Can! Energize our Families: A Family Guide.

40. Barlow SE. the Expert Committee. Expert Committee Recommendations Regarding the Prevention, Assessment, and Treatment of Child and Adolescent Overweight and Obesity: Summary Report. Pediatrics. 2007; 120:S164-S192. [PubMed: 18055651]

41. Rogers VW, Motyka E. 5-2-1-0 goes to school: A pilot project testing the feasibility of schools adopting and delivering healthy messages during the school day. Pediatrics. 2009; 123:S272S276. [PubMed: 19470603]

42. Wu, T.; Schetzina, K. Primary care based interventions on child overweight: a pilot study. Community Connections: Using Research Results to Reduce Health Disparities; Wintergreen, VA. June 25-28, 2007;

43. $\mathrm{BMi}^{2}$ Brief Motivational Interviewing to Reduce Body Mass Index Copyright 2009, The Regents of the University of Michigan. All rights reserved. Supported by the Centers for Disease Control and Prevention, Bristol-Myers Squibb Foundation, NHLBI Grant R01-HL085400.

44. Van Breukelen GJP. ANCOVA versus change from baseline had more power in randomized studies and more bias in nonrandomized studies. J Clin Epidemiol. 2006; 59:920-925. [PubMed: 16895814]

45. Pedhazur, EJ. Multiple regression in behavioral research: Explanation and prediction. 3. Fort Worth, TX: Harcourt Brace; 1997.

46. Raudenbush, SW.; Bryk, AS. Hierarchical linear models: Applications and data analysis methods. 2. Newbury Park, CA: Sage; 2002.

47. Allison, P. Fixed effects regression methods for longitudinal data using SAS. Cary, NC: SAS Institute Inc; 2005.

48. Cohen, J. Statistical power analysis for the behavioral sciences. 2. Hillsdale, NJ: Erlbaum; 1988.

49. Raudenbush SW, Liu X. Statistical power and optimal design for multisite randomized trials. Psychol Methods. 2000; 5:199-213. [PubMed: 10937329]

50. American Academy of Pediatrics (AAP). Policy Statement: Prevention of Overweight and Obesity. Pediatrics. 2003; 112:424-430. [PubMed: 12897303]

51. Futility FA. Avoidance medical professionals in the treatment of obesity. JAMA. 1993; 269:21323. [PubMed: 8468770] 
52. Kreuter MW, Schraff DP, Brennan LK, Lukwago SN. Physician recommendations for diet and physical activity: which patients get advised to change? Prev Med. 1997; 26:825-33. [PubMed: 9388794]

53. Schonfeld-Warden N, Warden C. Pediatric Obesity: an overview of etiology and treatment. Ped Endocrin. 1997; 44(2):339-354.

54. Logue, E.; Smucker, W., et al. Central Obesity and beliefs regarding diet, exercise, weight loss in a primary care setting. 22nd annual meeting of the North American Primary Care Research group; October 2-5, 1994; Toronto, Ontario, Canada.

55. Atkinson RL, Callaway CW, St Jeor S, Wolf-Novak L. A sane approach to weight loss. Patient Care. 1995 Nov.:152-80.

56. Story MT, et al. Management of Child and Adolescent Obesity: Attitudes, Barriers, Skills, and Training Needs Among Health Care Providers. Pediatrics. 2002; 110:210-214. [PubMed: 12093997]

57. Piatt GA, Orchard TJ, Emerson S, Simmons D, Songer TJ, Brooks MM, et al. Translating the chronic care model into the community: Results from a randomized controlled trial of a multifaceted diabetes care intervention. Diabetes Care. 2006; 29(4):811-817. [PubMed: 16567820]

58. Lozano P, Finkelstein JA, Carey VJ, Wagner EH, Inui TS, Fuhlbrigge AL, et al. A multisite randomized trial of the effects of physician education and organizational change in chronicasthma care: health outcomes of the Pediatric Asthma Care Patient Outcomes Research Team II Study. Arch Pediatr Adolesc Med. 2004; 158(9):875-83. [PubMed: 15351753]

59. Gaede P, Vedel P, Larsen N, Jensen GV, Parving HH, Pedersen O. Multifactorial intervention and cardiovascular disease in patients with type 2 diabetes. N Engl J Med. 2003; 348(5):383-393. [PubMed: 12556541]

60. Institute of Medicine. Crossing the Quality Chasm: A New Health System for the 21st Century. Mar 1. 2001

61. Miller, WR.; Rollnick, S. Motivational interviewing: Preparing people for change. 2. New York: The Guilford Press; 2002.

62. Rubak S, Sandboek, Lauritzen T, Christensen B. Motivational interviewing: A systematic review and meta-analysis. British Journal of General Practice. 2005; 55:305-312. [PubMed: 15826439]

63. Erickson SJ, Gerstle M, Feldstein SW. Brief interventions and motivational interviewing with children, adolescents, and their parents in pediatric health care settings. Arch Pediatr Adolesc Med. 2005; 159:1173-1180. [PubMed: 16330743]

64. Suarez M, Mullins S. Motivational interviewing and pediatric health behavior interventions. J Dev Behav Pediatr. 2008; 29:417-428. [PubMed: 18852613]

65. Resnicow K, Davis R, Rollnick S. Motivational interviewing for pediatric obesity: Conceptual issues and evidence review. J Am Diet Accoc. 2006; 106(12):2024-2033.

66. Schwartz RP, Hamre R, Dietz WH, Wasserman RC, Slora EJ, Myers EF, et al. Office-based motivational interviewing to prevent childhood obesity. Arch Pediatr Adolesc Med. 2007; 161:495-501. [PubMed: 17485627]

67. Foulkes, SH. Selected Papers of SH Foulkes: Psychoanalysis and Group Analysis. Foulkes, Elizabeth, editor. Karnac Books; 1990.

68. Renjilian D, Nezu A, Shermer R, Perri M, McKelvey W, Anton S. Individual versus group therapy for obesity. J Consult Clin Psychol. 2001; 69(4):717-721. [PubMed: 11550739]

69. ADA Evidence Analysis Library. How do group versus individual sessions compare in terms of effectiveness of treating childhood overweight? 2006.

70. American Academy of Pediatrics. AAP News. Sep.2001 19(3):93.

71. Anzman SL, Rollins BY, Birch LL. Parental influence on children's early eating environments and obesity risk: implications for prevention. Int J Obes. 2010; 34:1116-1124.

72. Trost SG, Sallis JF, Pate RR, Freedson PS, Taylor WC, Dowda M. Evaluating a model of parental influence on youth physical activity. Am J Prev Med. 2003; 25(4):277-282. [PubMed: 14580627]

73. Summerbell CD, Ashton V, Campbell KJ, Edmunds L, Kelly S, Waters E. Interventions for treating obesity in children (Cochrane Review). The Cochrane Library. 2004; (3) 
74. Trust for America's Health. (Retrieved 1/13/2005)

http://healthyamericans.org/reports/obesity2005/release.php?StateID=TN 


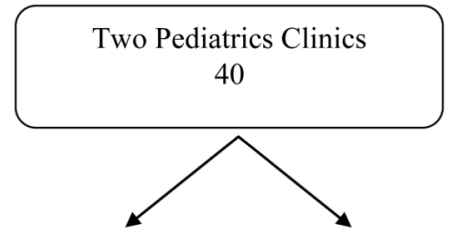

Two Family Practice Clinics 40
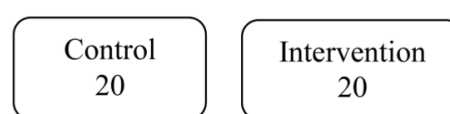
20

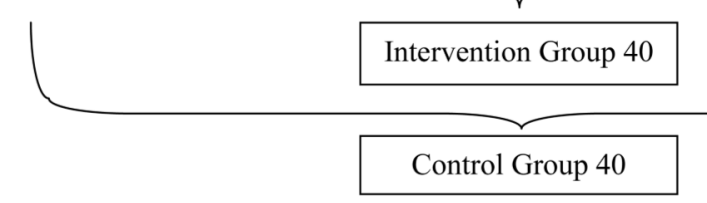

Figure 1.

Randomization procedures and number of children 


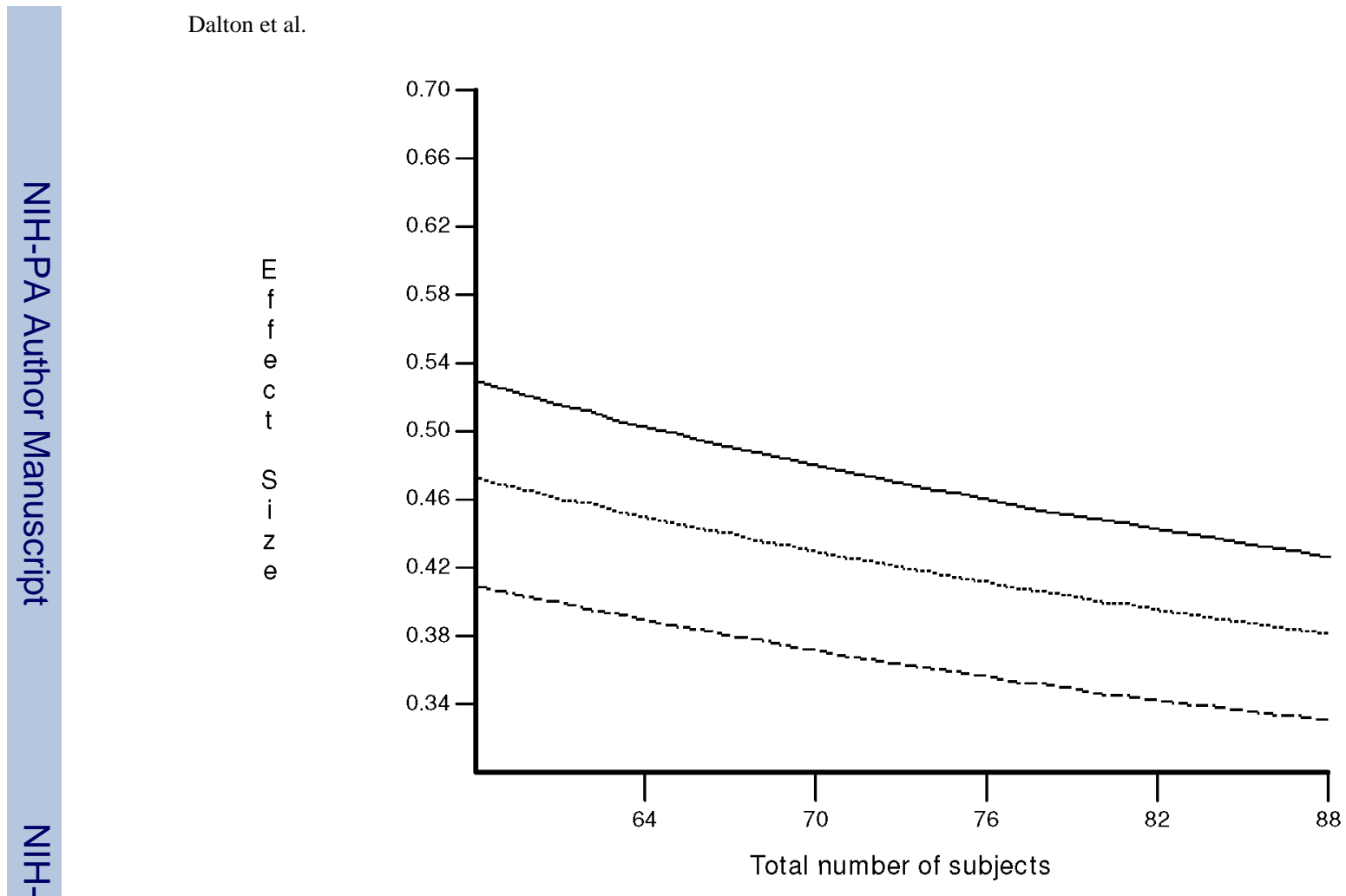

$\begin{aligned} \alpha & =0.050 \\ P & =0.80, R_{1}^{2}=0.50 \\ -P & =0.80, R_{1}^{2}{ }^{2}=0.60 \\ ---P & =0.80, R_{L 2}^{2}=0.70\end{aligned}$

Figure 2.

Sample size versus MDES 


\section{Table 1}

Schedule of data collection

\begin{tabular}{|c|c|c|c|c|}
\hline \multirow[t]{2}{*}{ Measure } & \multicolumn{2}{|c|}{ Screening/baseline } & \multicolumn{2}{|c|}{ Follow-up assessments } \\
\hline & Pre-screening & Month 0 & Month 3 & Month 6 \\
\hline Health Care Provider Questionnaire on Child Obesity & $\mathrm{X}$ & & & \\
\hline \multicolumn{5}{|l|}{ Identification of Eligible Children } \\
\hline - Child Height and Weight and BMI percentile & $\mathrm{X}$ & $\mathrm{X}$ & $\mathrm{X}$ & $\mathrm{X}$ \\
\hline Parent Consent & $\mathrm{X}$ & & & \\
\hline Child Assent & $\mathrm{X}$ & & & \\
\hline Children's Behavior Questionnaire & $\mathrm{X}$ & & & \\
\hline Raising Children Questionnaire & $\mathrm{X}$ & & & \\
\hline Healthy Eating and Physical Activity & $\mathrm{X}$ & & $\mathrm{X}$ & $\mathrm{X}$ \\
\hline \multicolumn{5}{|l|}{ Survey } \\
\hline \multicolumn{5}{|l|}{$\cdot \mathrm{CFQ}$} \\
\hline \multicolumn{5}{|l|}{ •FEAQ-R } \\
\hline \multicolumn{5}{|l|}{-Dietary Pattern Screen } \\
\hline \multicolumn{5}{|l|}{-DBS } \\
\hline Pediatric Quality of Life Inventory (Peds-QL) & $\mathrm{X}$ & & $\mathrm{X}$ & $\mathrm{X}$ \\
\hline \multicolumn{5}{|l|}{ Acceptability and Feasibility from the Parents Perspective } \\
\hline -We Can! Tell Us What You Think ${ }^{*}$ & & $\mathrm{X}$ & $\mathrm{X}$ & \\
\hline -We Can!Energize Our Families: Parent Program Participant Feedback Form * & & & $\mathrm{X}$ & \\
\hline
\end{tabular}

Note:

Month 3 is three months after the start of the intervention

CFQ (Child Feeding Questionnaire); FEAQ-R (Family Eating and Activity Habit Questionnaire); DBS (Dieting Belief Scales)

Indicates for intervention group only 
Table 2

Schedule of intervention components

\begin{tabular}{lll}
\hline Week & Format & Topic \\
\hline 1 & Group Session 1 & We Can! Energize our families: Getting started \\
2 & $\begin{array}{l}\text { Phone Follow-Up } \\
\text { Group Session } 2\end{array}$ & \\
3 & Phone Follow-Up * & We Can! Maintain a healthy weight: The energy balance equation \\
4 & Individual Visit 1 * & \\
5 & Group Session 3 & We Can! What to feed my family: Manage energy in \\
6 & Phone Follow-Up * & \\
7 & Group Session 4 & We Can! Less sit more fit: Energy out \\
8 & Phone Follow-Up * & \\
9 & Individual Visit 2** & \\
\hline
\end{tabular}

Note:

Individually tailored to family needs

** Indicates approximate timing of individual visit 
Table 3

Data safety monitoring plan

Item

- $\quad$ Baseline record of weight and general health will be created at the beginning of the study.

- During the intervention research staff will call each parent and ask if there are questions related to the information received, and how the parent plans to use this information.

- The project coordinator will call parents once a month and ask if there have been any problems related to the study. The project coordinator will also ask if there has been a change that is of concern to the parent (ex. Excess weight loss).

- $\quad$ Research Stall will meet and review information collected during the phone calls to identify any possible physical or psychological issues that may be of concern.

- If concerns are identified the appropriate member of the research team (clinical psychologist, parenting educator etc.) will contact the project participant and initiate appropriate measures to address the concern.

- $\quad$ All research staff will meet weekly for ongoing training, monitoring of protocol, and problem solving. Questions regarding data collection and safety issues are promptly reported to the primary investigator

- $\quad$ Research staff will be trained to identify events that would fall under mandatory reporting guidelines. These include physical injury to a child caused other than accidental means or information from a study participant that leads staff to believe a person is in imminent danger of physical harm.

- $\quad$ Research Staff will meet monthly for ongoing monitoring and problem solving.

- At the 6 month follow up the principal investigator and co-investigator will review data for possible problems. 